\title{
Determinants of anemia among pregnant women in northern Ghana
}

\author{
Martin N. Adokiya ${ }^{1,3^{*}}$, Richmond Aryeetey ${ }^{2}$, Monica Yost ${ }^{3}$, Andrew D. Jones ${ }^{4}$, Mark L. Wilson ${ }^{3}$
}

\section{Affiliations:}

$7 \quad{ }^{1}$ Department of Public Health, School of Allied Health Sciences, University for Development Studies, Ghana;

$8 \quad{ }^{2}$ Department of Population, Family and Reproductive Health, School of Public Health, University of Ghana,

9 Legon, Accra, Ghana

$10{ }^{3}$ Department of Epidemiology, School of Public Health, University of Michigan, Ann Arbor, United States of

11 America;

$12{ }^{4}$ Department of Nutritional Sciences, School of Public Health, University of Michigan, Ann Arbor, United States

13 of America

\section{Corresponding author}

16 Email: mnyaaba11@uds.edu.gh (MNA)

18 Running Head: Anemia in third trimester of pregnancy in northern Ghana

19 Funding: Funded under grant (D43TW009353) through the Fogarty International Center of the US National 20 Institutes of Health.

21 Financial Disclosure: The authors have no financial relationships to this article to disclose.

22 Conflict of Interest: The authors have no conflicts of interest relevant to this article to disclose. 


$\begin{array}{lll}23 & \text { Abbreviations } \\ 24 & \text { ANC } & \text { Antenatal Care } \\ 25 & \text { aOR } & \text { Adjusted Odds Ratio } \\ 26 & \text { CI } & \text { Confidence Interval } \\ 27 & \text { DHS } & \text { Demographic and Health Survey } \\ 28 & \text { FAO } & \text { Food and Agricultural Organization } \\ 29 & \text { GDP } & \text { Gross Domestic Product } \\ 30 & \text { GHS } & \text { Ghana Health Service } \\ 31 & \text { GHS } & \text { Ghana Cedis } \\ 32 & \text { GHSERC } & \text { Ghana Health Service Ethics Review Committee } \\ 33 & \text { Hb } & \text { Hemoglobin } \\ 34 & \text { HC } & \text { Health Centre } \\ 35 & \text { HF } & \text { Health Facility } \\ 36 & \text { HIV } & \text { Human Immuno-Deficiency Virus } \\ 37 & \text { IPT } & \text { Intermittent Preventive Treatment } \\ 38 & \text { ITN } & \text { Insecticide Treated Mosquito Net } \\ 39 & \text { MDD-W } & \text { Minimum Dietary Diversity for Women } \\ 40 & \text { OR } & \text { Odds Ratio } \\ 41 & \text { PCA } & \text { Principal Component Analysis } \\ 42 & \text { RCH } & \text { Reproductive and Child Health } \\ 43 & \text { TB } & \text { Tuberculosis } \\ 44 & \text { USD } & \text { United State Dollars }\end{array}$




\section{ABSTRACT}

2 Anemia is a global public health issue affecting half of all pregnant women in developing countries. In

$32014,42 \%$ of Ghanaian pregnant women aged $15-49$ years were anemic $(<11.0 \mathrm{~g} / \mathrm{dl})$ but information

4 on the determinants of anemia, particularly dietary diversity during the critical third trimester of

5 pregnancy is limited. We assessed the association between determinants and anemia among pregnant

6 women in northern Ghana.

7 We employed a cross-sectional design involving 624 pregnant women ( $\geq 20$ weeks of gestation)

8 attending four antenatal care (ANC) health facilities $\sim 25$ kilometres north of Tamale, Ghana between

9 July and August 2017. Hemoglobin concentration (measured using Hemocue HB 301) was classified

10 as severe, moderate, or mild. Other data included socio-demographic characteristics, malaria

11 prevention, deworming, and iron/folate tablet use. The FAO Minimum Dietary Diversity (MDD-W)

12 metric was used to categorize women into "inadequate" (MDD-W $<5$ food groups) and "adequate"

13 (MDD-W $\geq 5$ ). Logistic regression models were used to determine the association between

14 moderate/severe anemia $(\mathrm{Hb}<9.0 \mathrm{~g} / \mathrm{dl})$ and mild anemia $(9.0-10.9 \mathrm{~g} / \mathrm{dl})$, or with 'no anemia' $(\geq 11.0 \mathrm{~g} / \mathrm{dl})$

15 using STATA 14 software.

16 Of 624 women sampled, hemoglobin data were available for 601 . The mean age was $27.81 \pm 0.25$ years,

17 gestational age was $31.93 \pm 0.13$ weeks, ANC attendance was $3.89 \pm 0.07$; Hb concentration was

$189.73 \mathrm{~g} / \mathrm{dl} \pm 0.07$, and MDD-W index for ten food groups was $5.33 \pm 0.04$. Anemia $(\mathrm{Hb}<11.0 \mathrm{~g} / \mathrm{dl})$ was

19 observed in $74.8 \%$ of women (moderate/severe anemia $=33.4 \%$ and mild anemia $=41.4 \%$ ). Using

20 adjusted logistic regression, women who received deworming medication had lesser odds of being

21 moderate/severe anemic $(\mathrm{aOR}=0.51, \mathrm{P}=0.021)$. While women who were engaged in other occupation

22 (herdsmen) and no previous parity had higher odds of being moderate/severe anemic $(\mathrm{aOR}=2.90$,

$23 \mathrm{P}=0.042)$ and $(\mathrm{aOR}=2.13, \mathrm{P}=0.004)$ respectively. Moderate/severe anemia was not statistically

24 associated with MDD-W, nor with socioeconomic status/wealth index. Conclusion, anemia in 
25 pregnancy was nearly twice that of Ghana as a whole. Deworming medication was found to be

26 protective intervention for anemia during pregnancy.

27 Key words: Anemia, malaria, Diet Diversity, Pregnancy, Ghana

\section{INTRODUCTION}

Anemia is characterized by low blood hemoglobin $(\mathrm{Hb})$ concentration and constitutes an

32 important public health problem globally. Anemia has both short- and long-term consequences such as

33 preterm, low birth weight, morbidity and mortality 1,2,3,4. In 2016, World Health Organization (WHO)

34 estimated that anemia affected $38.2 \%$ of pregnant women globally, with the highest prevalence in

35 South-East Asia (48.7\%) and Africa (46.3\%) ${ }^{5,6}$. Anemia affects about 1.62 billion people, 56 million

36 of whom are pregnant women ${ }^{5}$. An estimated 800,000 pregnant women globally have severe anemia

$37(\mathrm{Hb}<7.0 \mathrm{~g} / \mathrm{dl})$. In Ghana, a national Demographic and Health Survey in 2014 determined that $42 \%$ of

38 pregnant women were anemic compared to $70 \%$ in rural parts of the country ${ }^{7}$.

40 Causes include environmental, behavioral, and social factors ${ }^{8}$ that limit adequate nutrient intake and

41 absorption, or exposure to infectious diseases. In addition, anemia risk is related to household-level

42 factors such as access to water and sanitation, availability of health services, access to diverse food

43 sources, use of insecticide treated nets (ITNs) and knowledge about anemia prevention. Other

44 household- or community-level factors include socioeconomic status, culture, wealth status and 45 education attainment $5,9,8$.

47 stores, making them especially vulnerable to iron deficiency anemia $6,10,11,12$. Hb concentration declines

48 during pregnancy, partly because of expanded plasma volume compared to red cell mass ${ }^{10,13}$. This is 
49 influenced partly by the iron status of the pregnant woman, ${ }^{12}$ representing a major public health

50 problem in sub-Saharan Africa ${ }^{9,10}$. Another contributor to anemia is parasitic infections/infestation

51 such as malaria, hookworm and schistosomiasis, especially in areas of Ghana where these infections

52 are endemic ${ }^{5}$. In addition, chronic infections such as tuberculosis (TB) and human immune-deficiency

53 virus (HIV) increase risk of anemia ${ }^{5}$. This condition may lead to premature delivery, intrauterine

54 growth retardation, and increased risk of malnutrition, morbidity and mortality for the mother, growing

55 fetus and newborn $10,11,14$.

Poor maternal diet during preconception and pregnancy is a major contributor to adverse

57 pregnancy outcome such as preterm, low birth weight, still birth and mortality. Diets of pregnant

58 women in developing countries are often limited to a few plant-based foods, with little consumption of

59 micronutrient-dense animal-source foods, or diverse fruits and vegetables ${ }^{13,15}$. Poor dietary intake and

60 low iron bioavailability are key determinants of low iron reserves and anemia ${ }^{16}$, particularly with little

61 dietary diversity among poor populations ${ }^{17}$, who consume mainly carbohydrates with little or no

62 animal products, fruits and vegetables ${ }^{10,18}$. Little is known about diet and anemia among pregnant

63 women in Ghana. Our study focused on northern Ghanaian women at high-risk of anemia in their third

64 trimester of pregnancy ${ }^{7}$. We aimed to assess the association between determinants, particularly dietary

65 diversity and moderate/severe anemia, with the hypothesis that, greater dietary diversity would be 66 associated with lower anemia risk.

\section{Materials and methods}

69 Study Area/Design

70 Ghana is a West African nation of 29 million people who are mostly concentrated in the

71 southern and coastal regions. Economically, Ghana ranks in the top third of African nations

$72(\mathrm{GDP} /$ capita $=$ US\$ 4,600), with considerable geographic variation in wealth. Our study was conducted 
73 in Northern Region, a poorer, predominantly agricultural area, made up of 28 Districts and

74 Municipalities. Four government antenatal care (ANC) health facilities in Savelugu Municipality,

75 located $\sim 25$ kilometres north of Tamale, served as the source of the study women. We employed a

76 cross-sectional design involving pregnant women ( $\geq 20$ weeks of gestation) seeking ANC, using our

77 own questionnaire and ANC medical record data to identify risk factors of anemia. The study was

78 undertaken during July-August 2017 (rainy season), using a questionnaire that had been pre-tested at a

79 nearby health center.

81 Study population and protection of human subjects

82 Data were collected from pregnant women attending health facilities in the North, South,

83 East and West quadrants of the Municipality. One health facility (HF) was randomly selected in the

84 South (Janjori Kukuo Health Centre) to pre-test the tools/questionnaire. Then, a HF was randomly

85 selected from each of the remaining three quadrants: Moglaa Health Centre (West), Savelugu

86 Reproductive and Child Health (East) and Pong Tamale Health Centre (North). The fourth HF sampled

87 was Savelugu Hospital, a major referral and health-seeking hospital centrally located in the

88 Municipality that serves many ANC-seeking women. During the ANC days of the four HFs, pregnant

89 women were recruited following informed consent. Women attending the ANC were informed of the

90 study in the native language, Dagbani, by a member of the study team. Women were eligible to

91 participate if they were at the HF to receive ANC, pregnant with a gestational age of at least 20 weeks,

9218 or more years of age, and had not been diagnosed with sickle cell anemia. The maternal records of

93 interested pregnant women were examined for compliance to these inclusion criteria. For eligible

94 women, a sticker with a unique identification number was placed on their maternal record book and

95 they were invited to stay for an interview. The women were then seen one-on-one with a trained

96 interviewer who explained the details of the study, including risks and benefits. Women who agreed to 
97 participate gave their consent via thumb print, and were then provided with a signed copy of their

98 consent form in English, as the native language is not commonly written, and many people are illiterate.

99 The study protocol was approved before its implementation by the Ghana Health Service

100 Ethical Review Committee (GHSERC/12/05/17) and the University of Michigan Institutional Review

101 Board for Health Sciences and Behavioral Sciences (HUM00128583). Additionally, official approval

102 letters were obtained from the Regional Director and District Director of Ghana Health Service in

103 Northern Region and Savelugu Municipality, respectively, as well as heads of the four HFs.

\section{Data sources and derived variables:}

Data were gathered from oral interviews using a pretested questionnaire, and from the ANC

record of each woman. The outcome of interest was the pregnant woman's anemia status. ANC is

mostly free to pregnant women in Ghana, and provides various interventions and preventative care to

111 intermittent preventive treatment of malaria during pregnancy (IPTp), deworming medication,

112 insecticide treated bed nets (ITN), and education about diet and malaria prevention ${ }^{7}$. While these

113 services are, in theory, widely available to all pregnant women, there are many factors influencing

114 whether a woman actually receives this care. Even though the ANC services are free, the transportation

115 to the clinic is not. Some women have to walk for many miles to reach the nearest ANC center. Rural

116 health centers are often poorly staffed and poorly supplied with materials and medications needed for

117 the services. Additionally, attending ANC may mean the loss of a day's income, which may not be

118 possible for lower-income women. Despite these challenges, a recent study reported that $97 \%$ of

119 pregnant Ghanaian women attended at least one ANC visit in 2014, and $87 \%$ had attended four or more

120 visits ${ }^{7}$. In the Northern Region where our study occurred, ANC attendance is likely to be lower due to 
121 lower income and fewer health centers. Even with high ANC attendance, anemia remains a major

122 problem for adult women in many regions of Ghana, particularly in areas like our study sites where

123 poverty levels are high, and access to health centers is hindered ${ }^{7}$.

124 The interviews were conducted by trained local research assistants with health education

125 backgrounds (e.g. Bachelor of Science degree in Nursing or Community Nutrition). Each hired research

126 assistant was fluent in the Dagbani and English languages, as well as other local native Ghanaian

127 languages. The interviews were conducted in a private room or area of the HF to protect women's

128 privacy. Individual women were asked questions concerning their demographic situation, as well as

129 characteristics of their housing, water, toilets, and household assets. In addition, they were asked about

130 the ANC services they had received, and were administered a 24-hour dietary recall survey, collecting

131 information on all food items and beverages consumed in the previous day. Interviewers asked

132 individual women to recall all foods they had consumed in the previous 24-hour, and after responding

133 were probed to ensure that no meal or snack was left out (breakfast, snack before lunch, lunch, snack

134 after lunch, dinner and snack before going to bed). The foods were then categorized into ten (10) food

135 groups ${ }^{19}$. Information on $\mathrm{Hb}$ concentration, and gestation, were extracted from the maternal health

136 records that are kept for each pregnant woman.

$137 \mathrm{Hb}$ concentration determination differed between the HFs. The Savelugu hospital used a

138 spectrophotometer operated by trained laboratory scientist/technicians. The three health centers used a

139 Hemocue HB 301 operated by HF workers. A blood sample for Hb concentration was taken on the day

140 of the interview in most cases, but for very few participants this occurred one week after the interview.

141 Our study used results from tests that are routinely conducted at ANC visits, as recommended

142 by the Government of Ghana. All tests were performed by HF staff. Occasionally, tests were not

143 performed on the day of interview due to shortages of supplies. However, tests for these women were

144 completed in the following weeks if they returned to the same HF. Overall, 27 women did not return 
145 for testing. After completing the interview and data extraction, participants were given GHS10.00 (US\$

$1462.27)$ to compensate them for transportation back to their homes.

\section{Statistical analysis:}

150 to describe the distribution of anemia status and all hypothesized explanatory variables. Logistic

151 regression was performed to determine the unadjusted associations between anemia status and each

152 independent explanatory variable. Adjusted logistic regression analyses were performed to evaluate

153 relationships among independent factors and anemia status. As with the Demographic and Health

154 Survey's (DHS) classification scheme, we defined anemia as severe $(\mathrm{Hb}<7.0 \mathrm{~g} / \mathrm{dl})$, moderate $(\mathrm{Hb}=7.0$ -

$1558.9 \mathrm{~g} / \mathrm{dl})$, or mild $(\mathrm{Hb}=9.0-10.9 \mathrm{~g} / \mathrm{dl})$, with no anemia being $\mathrm{Hb} \geq 11.0 \mathrm{~g} / \mathrm{dl}$. This cut-off was selected in

156 accordance with the Demographic and Health Survey for Ghana and previous studies of anemia in

157 pregnant women $3,7,10$. For our study, severe and moderate anemia were combined (moderate/severe)

158 as $\mathrm{Hb}<9.0 \mathrm{~g} / \mathrm{dl}$, and analyzed in comparison with mild/no anemia. Anemia prevalence was analyzed

159 for the entire study population, as well as for each HF, using logistic regression tests to evaluate risk

160 factor associations.

161 Dietary diversity was calculated using the reported number of different food groups consumed

162 by each woman in the previous 24 hours. Food was reclassified into ten distinct food groups: (1) grains,

163 roots and tubers, (2) Pulses-beans, peas and lentils, (3) Nuts and seeds, (4) Dairy, (5) Meat, poultry and

164 fish, (6) Eggs, (7) Dark green leafy vegetables, (8) Vitamin A-rich fruits and vegetables, (9) Other

165 vegetables and (10) Other fruits. These mutually exclusive food groups are those that comprise the

166 FAO Minimum Dietary Diversity for Women (MDD-W) indicator ${ }^{19}$. The FAO MDD-W metric was

167 used to categorize participants into "inadequate" (MDD-W $<5$ food groups) and "adequate" (MDD-W $168 \geq 5$ ). A wealth index was calculated using Principal Component Analysis (PCA) of household assets, 
169 housing conditions, water facilities and toilet facilities. Other risk factors that were analyzed included

170 maternal education, religion, ethnicity, and occupation as well as socio-demographic characteristics,

171 ANC attendance, parity, ITN ownership and ITN utilization. The relation between each individual risk

172 factor and moderate/severe anemia was determined using bivariate logistic regression to obtain the

173 odds ratios (ORs), confidence intervals (CIs) and probability values (p-value) for the whole sample.

174 Separate adjusted logistic regression models were constructed for the whole sample using ten (10)

175 selected risk factors with a significance test level of alpha $=0.05$. Odds ratios were used to interpret

176 the associations of risk factors with moderate/severe or mild anemia/no anemia.

178 RESULTS

179 Study population characteristics:

180 In total, 624 pregnant women at $\geq 20$ weeks of gestation were enrolled, but analysis was limited

181 to 601 participants $(96.3 \%)$ for whom $\mathrm{Hb}$ measurements were available (Table 1). The mean age was

$18227.81( \pm 0.25)$ years, with gestation age $=31.93( \pm 0.13), \mathrm{ANC}=3.89( \pm 0.07), \mathrm{Hb}=9.73( \pm 0.07)$ and

183 MDD-W $=5.33( \pm 0.04)$. More than half $(58 \%)$ of the participants who received ANC services at the

184 Savelugu Hospital and 53\% were between 20 and 29 years old. Nearly all women (96\%) were from the

185 Dagomba ethnic group. Three-quarters (75\%) had no formal education, and nearly all (97\%) belonged

186 to the Islamic religion, with $98 \%$ being married (monogamous or polygamous marriages). About $69 \%$

187 of the participants were either farmers $(39 \%)$ or petty traders $(30 \%)$, while very few $(2 \%)$ were salaried

188 workers. Most women (91\%) were between 28 and 36 weeks of gestation when interviewed, while

189 very few (2\%) were between 20 and 27 weeks of gestation. Approximately $92 \%$ of the participants had

190 access to improved water sources and about one-quarter (24\%) reported using improved toilet facilities.

About $85 \%$ of the participants achieved the MDD-W indicator, that is, consumed five or more

192 food groups in the previous 24 hours. In addition, 91\% of the participants reported household 
193 ownership of at least one ITN, with $83 \%$ having slept under an ITN on the night preceding the

194 interview. About three-quarters (76\%) of the participants had previously delivered live babies. While

$19552 \%$ had attended $\geq 4$ ANC visits and $84 \%$ received at least one IPTp treatment, only $14 \%$ received any

196 deworming medication. In addition, 99\% of the participants reported they had received iron and folic 197 acid tablets during ANC visits.

\section{Prevalence of moderate/severe anemia and risk factors}

200 Three-quarters (75\%) of the pregnant women in the study were anemic with $41 \%$ experiencing

201 mild anemia and 33\% experiencing moderate/severe anemia. Forty-one percent of participants who

202 were $<20$ years of age had moderate/severe anemia. Anemia prevalence declined with increasing age.

203 Moderate/severe anemia was least prevalent among salaried workers (29\%) and petty traders (30\%),

204 but highest among unemployed (41\%) and others, such as herdsmen (63\%).

Nearly half (49\%) of the participants without household ownership of ITNs were

206 moderate/severe anemic compared to $32 \%$ among those with ITNs. Those who did not sleep under ITN

207 on the previous night preceding the survey were more likely to experience moderate/severe anemia

208 (44\%) compared to those who slept under an ITN (31\%). In addition, 41\% of the participants who did

not receive Intermittent Preventive Treatment (IPT) had moderate/severe anemia compared to $32 \%$ in

210 those who did receive IPT.

211 Women with no previous delivery had the greater risk of moderate/severe anemia (48\%)

212 compared to women with at least one previous deliveries. More than a third of pregnant women who

213 did not receive deworming medication had moderate/severe anemia compared to about one-fifth among

214 those who did take deworming drugs. Similarly, about 33\% of the participants who received iron tablets

215 or folic acid tablets did not have moderate/severe anemia (Table 1). 
217 (36\%), dark green leafy vegetables (34\%), and other vegetables (34\%) had greater risk of

218 moderate/severe anemia than those who did not. However, participants who consumed nuts/seeds

$219(33 \%)$, vitamin A-rich fruits and vegetables (20\%) and other fruits $(30 \%)$ were less likely to have

220 moderate/severe anemia compared to those who did not consume.

221

Table 1: Socio-demographic factors and dietary diversity by anemia status among pregnant women in northern Ghana $(n=601)$

\begin{tabular}{|c|c|c|c|c|}
\hline Variables & $\begin{array}{l}\text { Total Sample } \\
(\mathrm{N}=601)\end{array}$ & $\begin{array}{c}\text { Moderate/severe } \\
\text { anemia } \\
(<9.0 \mathrm{~g} / \mathrm{dl})\end{array}$ & $\begin{array}{l}\text { Mild anemia } \\
(9.0-10.9 \mathrm{~g} / \mathrm{dl})\end{array}$ & $\begin{array}{l}\text { No anemia } \\
(\geq 11.0 \mathrm{~g} / \mathrm{dl})\end{array}$ \\
\hline & Frequency (\%) & Frequency (\%) & Frequency (\%) & Frequency (\%) \\
\hline $\begin{array}{l}\text { Name of Health Facility } \\
\text { Savelugu hospital } \\
\text { Moglaa HC } \\
\text { Pong Tamale HC } \\
\text { Savelugu RCH }\end{array}$ & $\begin{array}{l}349(58.1) \\
93(15.5) \\
77(12.8) \\
82(13.6)\end{array}$ & $\begin{array}{l}157(45.0) \\
14(15.0) \\
18(23.4) \\
12(14.6)\end{array}$ & $\begin{array}{l}108(31.0) \\
54(58.1) \\
44(57.1) \\
43(52.4)\end{array}$ & $\begin{array}{l}84(24.0) \\
25(26.9) \\
15(19.5) \\
27(33.0)\end{array}$ \\
\hline $\begin{array}{l}\text { Age (in years) } \\
<20 \\
20-29 \\
30-39 \\
\geq 40\end{array}$ & $\begin{array}{c}34(5.7) \\
319(53.1) \\
226(37.6) \\
22(3.6)\end{array}$ & $\begin{array}{l}14(41.2) \\
112(35.1) \\
73(32.3) \\
2(9.1)\end{array}$ & $\begin{array}{l}15(44.1) \\
125(39.2) \\
95(42.0) \\
14(63.6)\end{array}$ & $\begin{array}{l}5(14.7) \\
82(25.7) \\
58(25.7) \\
6(27.3)\end{array}$ \\
\hline $\begin{array}{l}\text { Ethnicity } \\
\text { Dagomba } \\
\text { Fulani } \\
\text { Others (Akan, Ewe etc.) }\end{array}$ & $\begin{array}{c}576(96.2) \\
8(1.3) \\
15(2.5) \\
\end{array}$ & $\begin{array}{c}195(33.8) \\
2(25.0) \\
3(20.0) \\
\end{array}$ & $\begin{array}{c}236(41.0) \\
5(62.5) \\
7(46.7) \\
\end{array}$ & $\begin{array}{c}145(25.2) \\
1(12.5) \\
5(33.3) \\
\end{array}$ \\
\hline $\begin{array}{l}\text { Education status } \\
\text { None } \\
\text { Primary } \\
\text { JSS/Middle School } \\
\text { Senior Secondary } \\
\text { Tertiary } \\
\end{array}$ & $\begin{array}{c}450(74.9) \\
46(7.7) \\
53(8.8) \\
38(6.3) \\
14(2.3) \\
\end{array}$ & $\begin{array}{l}139(30.9) \\
19(41.3) \\
22(41.5) \\
17(44.7) \\
4(28.6) \\
\end{array}$ & $\begin{array}{c}204(45.3) \\
16(34.8) \\
15(28.3) \\
11(29.0) \\
3(21.4) \\
\end{array}$ & $\begin{array}{c}107(23.8) \\
11(23.9) \\
16(30.2) \\
10(26.3) \\
7(50.0) \\
\end{array}$ \\
\hline $\begin{array}{l}\text { Religious denomination } \\
\text { Islam } \\
\text { Christianity } \\
\text { Traditionalist }\end{array}$ & $\begin{array}{c}582(97.3) \\
13(2.2) \\
3(0.50) \\
\end{array}$ & $\begin{array}{c}196(33.7) \\
4(30.8) \\
0(0.0)\end{array}$ & $\begin{array}{c}238(40.9) \\
7(53.8) \\
2(66.7) \\
\end{array}$ & $\begin{array}{c}148(25.4) \\
2(15.4) \\
1(33.3)\end{array}$ \\
\hline $\begin{array}{l}\text { Marital status } \\
\text { Single } \\
\text { Married-monogamous } \\
\text { Married-polygamous }\end{array}$ & $\begin{array}{c}12(2.0) \\
329(54.7) \\
260(43.3) \\
\end{array}$ & $\begin{array}{c}5(41.7) \\
103(31.3) \\
93(35.8) \\
\end{array}$ & $\begin{array}{c}7(58.3) \\
139(42.3) \\
103(39.6) \\
\end{array}$ & $\begin{array}{c}0(0.0) \\
87(26.4) \\
64(24.6) \\
\end{array}$ \\
\hline $\begin{array}{l}\text { Occupation status } \\
\text { Farmer } \\
\text { Petty trader } \\
\text { Salaried worker } \\
\text { Artisan/laborer }\end{array}$ & $\begin{array}{c}233(38.8) \\
181(30.1) \\
14(2.3) \\
79(13.1)\end{array}$ & $\begin{array}{c}73(31.3) \\
54(29.9) \\
4(28.6) \\
27(34.2)\end{array}$ & $\begin{array}{l}108(46.4) \\
73(40.3) \\
4(28.6) \\
34(43.0)\end{array}$ & $\begin{array}{c}52(22.3) \\
54(29.8) \\
6(42.8) \\
18(22.8)\end{array}$ \\
\hline
\end{tabular}




\begin{tabular}{|c|c|c|c|c|}
\hline $\begin{array}{l}\text { No occupation } \\
\text { Others }\end{array}$ & $\begin{array}{c}75(12.5) \\
19(3.2) \\
\end{array}$ & $\begin{array}{l}31(41.3) \\
12(63.2) \\
\end{array}$ & $\begin{array}{c}27(36.0) \\
3(15.8) \\
\end{array}$ & $\begin{array}{c}17(22.7) \\
4(21.0)\end{array}$ \\
\hline $\begin{array}{l}\text { Wealth index } \\
\text { Poorest } \\
\text { Poor } \\
\text { Medium } \\
\text { Wealthy } \\
\text { Wealthiest }\end{array}$ & $\begin{array}{l}118(19.6) \\
119(19.8) \\
120(20.0) \\
122(20.3) \\
122(20.3)\end{array}$ & $\begin{array}{l}34(28.8) \\
41(37.5) \\
45(37.5) \\
38(31.1) \\
43(35.2)\end{array}$ & $\begin{array}{l}53(44.9) \\
50(42.0) \\
51(42.5) \\
45(36.9) \\
50(41.0)\end{array}$ & $\begin{array}{l}31(26.3) \\
28(23.5) \\
24(20.0) \\
39(32.0) \\
29(23.8)\end{array}$ \\
\hline $\begin{array}{l}\text { Gestational age (in weeks) } \\
20-27 \\
28-36 \\
\geq 37\end{array}$ & $\begin{array}{c}11(1.8) \\
547(91.0) \\
43(7.2)\end{array}$ & $\begin{array}{c}4(36.4) \\
182(33.2) \\
15(34.9)\end{array}$ & $\begin{array}{c}4(36.4) \\
229(41.9) \\
16(37.2)\end{array}$ & $\begin{array}{c}3(27.2) \\
136(24.9) \\
12(27.9)\end{array}$ \\
\hline $\begin{array}{l}\text { Drinking water source } \\
\text { Non-improved } \\
\text { Improved }\end{array}$ & $\begin{array}{c}48(8.0) \\
553(92.0)\end{array}$ & $\begin{array}{c}12(25.0) \\
189(34.2)\end{array}$ & $\begin{array}{c}25(52.1) \\
224(40.5)\end{array}$ & $\begin{array}{c}11(22.9) \\
140(25.3)\end{array}$ \\
\hline $\begin{array}{l}\text { Toilet facility } \\
\text { Non-improved } \\
\text { Improved }\end{array}$ & $\begin{array}{l}457(76.0) \\
144(24.0)\end{array}$ & $\begin{array}{l}155(33.9) \\
46(31.94)\end{array}$ & $\begin{array}{l}185(40.5) \\
64(44.44)\end{array}$ & $\begin{array}{l}117(25.6) \\
34(23.61)\end{array}$ \\
\hline $\begin{array}{l}\text { Grains/roots/tubers/plantain } \\
\text { No } \\
\text { Yes }\end{array}$ & $\begin{array}{c}1(0.2) \\
600(99.8)\end{array}$ & & & \\
\hline $\begin{array}{l}\text { Pulses } \\
\text { No } \\
\text { Yes }\end{array}$ & $\begin{array}{l}452(75.3) \\
148(24.7)\end{array}$ & $\begin{array}{c}139(30.8) \\
61(41.2)\end{array}$ & $\begin{array}{c}194(42.9) \\
55(37.2)\end{array}$ & $\begin{array}{c}119(26.3) \\
32(21.6)\end{array}$ \\
\hline $\begin{array}{l}\text { Nuts/seeds } \\
\text { No } \\
\text { Yes } \\
\end{array}$ & $\begin{array}{l}133(22.2) \\
467(77.8) \\
\end{array}$ & $\begin{array}{c}48(36.1) \\
153(32.8)\end{array}$ & $\begin{array}{c}53(39.8) \\
195(41.7) \\
\end{array}$ & $\begin{array}{c}32(24.1) \\
119(25.5) \\
\end{array}$ \\
\hline $\begin{array}{l}\text { Dairy } \\
\text { No } \\
\text { Yes } \\
\end{array}$ & $\begin{array}{l}477(79.4) \\
124(20.6)\end{array}$ & $\begin{array}{c}158(33.1) \\
43(34.7)\end{array}$ & $\begin{array}{c}197(41.3) \\
52(41.9)\end{array}$ & $\begin{array}{c}122(25.6) \\
29(23.4)\end{array}$ \\
\hline $\begin{array}{l}\text { Meat/poultry/fish(amaani) } \\
\text { No } \\
\text { Yes }\end{array}$ & $\begin{array}{c}7(1.2) \\
594(98.8)\end{array}$ & $\begin{array}{c}2(28.6) \\
199(33.5)\end{array}$ & $\begin{array}{c}3(42.8) \\
246(41.4)\end{array}$ & $\begin{array}{c}2(28.6) \\
149(25.1)\end{array}$ \\
\hline $\begin{array}{l}\text { Eggs } \\
\text { No } \\
\text { Yes }\end{array}$ & $\begin{array}{c}555(92.5) \\
45(7.5)\end{array}$ & $\begin{array}{c}185(33.3) \\
16(35.6)\end{array}$ & $\begin{array}{c}233(42.0) \\
16(35.6)\end{array}$ & $\begin{array}{c}137(24.7) \\
13(28.8)\end{array}$ \\
\hline $\begin{array}{l}\text { Dark green leafy vegetables } \\
\text { No } \\
\text { Yes }\end{array}$ & $\begin{array}{c}40(6.7) \\
561(93.3)\end{array}$ & $\begin{array}{c}10(25.0) \\
191(34.0)\end{array}$ & $\begin{array}{c}22(55.0) \\
227(40.5)\end{array}$ & $\begin{array}{c}8(20.0) \\
143(25.5)\end{array}$ \\
\hline $\begin{array}{llll}\text { Vitamin } & \text { A-rich } & \text { fruits } & \& \\
\text { vegetables } & & & \\
\text { No } & & & \\
\text { Yes } & & & \\
\end{array}$ & $\begin{array}{c}555(92.3) \\
46(7.7)\end{array}$ & $\begin{array}{c}192(34.6) \\
9(19.6)\end{array}$ & $\begin{array}{c}223(40.2) \\
26(56.5)\end{array}$ & $\begin{array}{c}140(25.2) \\
11(23.9)\end{array}$ \\
\hline $\begin{array}{l}\text { Other vegetables } \\
\text { No } \\
\text { Yes }\end{array}$ & $\begin{array}{c}11(1.8) \\
590(98.2)\end{array}$ & $\begin{array}{c}3(27.3) \\
198(33.6)\end{array}$ & $\begin{array}{c}6(54.5) \\
243(41.2)\end{array}$ & $\begin{array}{c}2(18.2) \\
149(25.2)\end{array}$ \\
\hline $\begin{array}{l}\text { Other fruits } \\
\text { No } \\
\text { Yes }\end{array}$ & $\begin{array}{c}570(95.0) \\
30(5.0)\end{array}$ & $\begin{array}{c}191(33.5) \\
9(30.0)\end{array}$ & $\begin{array}{c}234(41.1) \\
15(50.0)\end{array}$ & $\begin{array}{c}145(25.4) \\
6(20.0)\end{array}$ \\
\hline $\begin{array}{l}\text { Minimum dietary diversity } \\
\text { score }\end{array}$ & & & & \\
\hline
\end{tabular}


bioRxiv preprint doi: https://doi.org/10.1101/708784; this version posted July 20, 2019 . The copyright holder for this preprint (which was not certified by peer review) is the author/funder. This article is a US Government work. It is not subject to copyright under 17 USC 105 and is also made available for use under a CCO license.

\begin{tabular}{|c|c|c|c|c|}
\hline $\begin{array}{l}<5 \\
\geq 5\end{array}$ & $\begin{array}{c}91(15.1) \\
510(84.9)\end{array}$ & $\begin{array}{c}27(29.7) \\
174(34.1)\end{array}$ & $\begin{array}{c}40(43.9) \\
209(41.0)\end{array}$ & $\begin{array}{c}24(26.4) \\
127(24.9)\end{array}$ \\
\hline $\begin{array}{l}\text { Household livestock } \\
\text { No } \\
\text { Yes }\end{array}$ & $\begin{array}{l}116(19.3) \\
485(80.7)\end{array}$ & $\begin{array}{c}36(31.0) \\
165(34.0)\end{array}$ & $\begin{array}{c}45(38.8) \\
204(42.1)\end{array}$ & $\begin{array}{c}35(30.2) \\
116(23.9)\end{array}$ \\
\hline $\begin{array}{l}\text { Household ITN } \\
\text { No } \\
\text { Yes }\end{array}$ & $\begin{array}{c}55(9.2) \\
546(90.8)\end{array}$ & $\begin{array}{c}27(49.1) \\
174(31.9)\end{array}$ & $\begin{array}{c}17(30.9) \\
232(42.5)\end{array}$ & $\begin{array}{c}11(20.0) \\
140(25.6)\end{array}$ \\
\hline $\begin{array}{l}\text { Slept under ITN previous night } \\
\text { No } \\
\text { Yes }\end{array}$ & $\begin{array}{l}104(17.3) \\
497(82.7)\end{array}$ & $\begin{array}{c}46(44.2) \\
155(31.2)\end{array}$ & $\begin{array}{c}35(33.7) \\
214(43.1) \\
\end{array}$ & $\begin{array}{c}23(22.1) \\
128(25.7)\end{array}$ \\
\hline $\begin{array}{l}\text { No. of previous deliveries } \\
0 \\
1 \\
2-3 \\
\geq 4\end{array}$ & $\begin{array}{l}142(23.6) \\
122(20.3) \\
214(35.6) \\
123(20.5)\end{array}$ & $\begin{array}{l}68(47.9) \\
40(32.8) \\
59(27.6) \\
34(27.7)\end{array}$ & $\begin{array}{l}48(33.8) \\
54(44.3) \\
91(42.5) \\
56(45.5)\end{array}$ & $\begin{array}{l}26(18.3) \\
28(22.9) \\
64(29.9) \\
33(26.8)\end{array}$ \\
\hline $\begin{array}{l}\text { Total ANC visits } \\
<4 \\
4-7 \\
\geq 8\end{array}$ & $\begin{array}{l}286(47.6) \\
286(47.6) \\
29(4.8)\end{array}$ & $\begin{array}{c}97(33.9) \\
96(33.6) \\
8(27.6)\end{array}$ & $\begin{array}{c}123(43.0) \\
111(38.8) \\
15(51.7)\end{array}$ & $\begin{array}{c}66(23.1) \\
79(27.6) \\
6(20.7)\end{array}$ \\
\hline $\begin{array}{l}\text { IPT received } \\
\text { No } \\
\text { Yes }\end{array}$ & $\begin{array}{c}99(16.5) \\
502(83.5)\end{array}$ & $\begin{array}{c}41(41.4) \\
160(31.9)\end{array}$ & $\begin{array}{c}38(38.4) \\
211(42.0)\end{array}$ & $\begin{array}{c}20(20.2) \\
131(26.1)\end{array}$ \\
\hline $\begin{array}{l}\text { Deworming medication } \\
\text { No } \\
\text { Yes }\end{array}$ & $\begin{array}{c}515(85.7) \\
86(14.3)\end{array}$ & $\begin{array}{c}183(35.5) \\
18(20.9)\end{array}$ & $\begin{array}{c}198(38.5) \\
51(59.3)\end{array}$ & $\begin{array}{c}134(26.0) \\
17(19.8)\end{array}$ \\
\hline $\begin{array}{l}\text { Received iron tablets } \\
\text { No } \\
\text { Yes }\end{array}$ & $\begin{array}{c}3(0.5) \\
598(99.5)\end{array}$ & $\begin{array}{c}1(33.3) \\
200(33.4)\end{array}$ & $\begin{array}{c}1(33.3) \\
248(41.5)\end{array}$ & $\begin{array}{c}1(33.3) \\
150(25.1)\end{array}$ \\
\hline $\begin{array}{l}\text { Received folic acid } \\
\text { No } \\
\text { Yes }\end{array}$ & $\begin{array}{c}3(0.5) \\
598(99.5)\end{array}$ & $\begin{array}{c}1(33.3) \\
200(33.4)\end{array}$ & $\begin{array}{c}1(33.3) \\
248(41.5)\end{array}$ & $\begin{array}{c}1(33.3) \\
150(25.1)\end{array}$ \\
\hline $\begin{array}{l}\text { Prevalence of anemia } \\
\text { Severe-moderate }(<9.0 \mathrm{~g} / \mathrm{dl}) \\
\text { Mild }(9.0-10.9 \mathrm{~g} / \mathrm{dl}) \\
\text { No anemia }\end{array}$ & $\begin{array}{l}201(33.5) \\
249(41.4) \\
151(25.1) \\
\end{array}$ & & & \\
\hline $\begin{array}{l}\text { Prevalence of anemia types } \\
\text { Severe }(<7.0 \mathrm{~g} / \mathrm{dl}) \\
\text { Moderate }(7.0-8.9 \mathrm{~g} / \mathrm{dl}) \\
\text { Mild }(9.0-10.9 \mathrm{~g} / \mathrm{dl}) \\
\text { No anemia }(\geq 11.0 \mathrm{~g} / \mathrm{dl}) \\
\end{array}$ & $\begin{array}{c}25(4.2) \\
175(29.2) \\
249(41.5) \\
151(25.1) \\
\end{array}$ & & & \\
\hline $\begin{array}{l}\text { Means } \\
\text { Age (in years) } \\
\text { Gestational age (in weeks) } \\
\text { Number of ANC visits } \\
\text { Hemoglobin level } \\
\text { Minimum Dietary diversity }\end{array}$ & $\begin{array}{c}\text { Mean (CI) } \\
\\
27.81(27.33,28.29) \\
31.93(31.68,32.18) \\
3.89(3.76,4.03) \\
9.73(9.59,9.88) \\
5.33(5.25,5.42) \\
\end{array}$ & $\begin{array}{c}\text { Standard } \\
\text { Error } \\
0.246 \\
0.128 \\
0.070 \\
0.074 \\
0.042 \\
\end{array}$ & & \\
\hline
\end{tabular}


$(\mathbf{O R}=\mathbf{0 . 2 1}, 95 \% \mathrm{CI}: 0.11,0.40)$. Moderate/severe anemia was less frequent among women who were moderate/severe anemia than those who did not receive $(\mathbf{O R}=\mathbf{0 . 4 8}, 95 \% \mathrm{CI}: 0.28,0.83)$. Interestingly,

241 moderate/severe anemia was more likely among women who were engaged in other occupations (e.g.

242 herdsmen) $(\mathbf{O R}=\mathbf{3 . 7 6}, 95 \% \mathrm{CI}: 1.42,9.94)$, or who consumed pulses $(\mathbf{O R}=\mathbf{1 . 5 8}, 95 \% \mathrm{CI}: 1.06,2.32)$.

244 ITN and reported sleeping under an ITN. Women who did not own ITN were more than twice as likely

245 to be moderate/severe anemic $(\mathbf{O R}=\mathbf{2 . 0 6}, 95 \% \mathrm{CI}: 1.18,3.60)$, while women who reported not sleeping

246 under an ITN the previous night had almost twice the risk of being moderate/severe anemic $(\mathbf{O R}=\mathbf{1 . 7 5}$,

$24795 \% \mathrm{CI}: 1.14,2.69)$. Those women who had not previously delivered were more than twice as likely to

248 be moderate/severe anemic compared to women with one or more births ( $\mathrm{OR}=\mathbf{2 . 4 1}, 95 \% \mathrm{CI}: 1.55,3.77)$.

249 None of the remaining hypothesized risk factors, such as socioeconomic status, minimum dietary 
bioRxiv preprint doi: https://doi.org/10.1101/708784; this version posted July 20,2019 . The copyright holder for this preprint (which was not certified by peer review) is the author/funder. This article is a US Government work. It is not subject to copyright under 17 USC 105 and is also made available for use under a CCO license.

250 diversity, gestational age, and number of ANC visits, were significantly associated with 251 moderate/severe anemia. 
Table 2: Bivariate odd ratios for anemia status among pregnant women in Northern Ghana $(\mathrm{n}=601)$

\begin{tabular}{|c|c|c|c|c|c|c|c|c|c|}
\hline & & $\begin{array}{l}\text { Anemia } \\
(<11.0 \mathrm{~g} / \mathrm{dl})\end{array}$ & $\begin{array}{c}\text { No } \\
\text { anemia } \\
(\geq 11.0 \mathrm{~g} / \mathrm{dl}\end{array}$ & $\begin{array}{l}\text { Anemia }(<11.0 \mathrm{~g} / \mathrm{dl}) \\
\text { no anemia }(\geq 11.0 \mathrm{~g} / \mathrm{d}\end{array}$ & & $\begin{array}{l}\text { Moderate/ } \\
\text { severe } \\
\text { anemia }\end{array}$ & $\begin{array}{l}\text { Mild } / \text { no } \\
\text { anemia } \\
(\geq 9.0 \mathrm{~g} / \mathrm{dl}\end{array}$ & $\begin{array}{l}\text { Moderate sever } \\
(<9.0 \mathrm{~g} / \mathrm{dl}) \text { versu } \\
\text { anemia }(\geq 9.0\end{array}$ & $\begin{array}{l}\text { nemia } \\
\text { nild/no } \\
\text { dl) }\end{array}$ \\
\hline & Frequency & $\%$ & $\%$ & unadjusted OR(CI) & $\begin{array}{c}\mathrm{P}- \\
\text { value }\end{array}$ & $\%$ & $\%$ & $\begin{array}{l}\text { unadjusted } \\
\text { OR(CI) }\end{array}$ & P-value \\
\hline $\begin{array}{l}\text { Health Facility } \\
\text { Savelugu hospital } \\
\text { Moglaa HC } \\
\text { Pong Tamale HC } \\
\text { Savelugu RCH } \\
\end{array}$ & $\begin{array}{c}349 \\
93 \\
77 \\
82 \\
\end{array}$ & $\begin{array}{l}75.9 \\
73.1 \\
80.5 \\
67.1 \\
\end{array}$ & $\begin{array}{l}24.1 \\
26.9 \\
19.5 \\
32.9 \\
\end{array}$ & $\begin{array}{c}\text { Ref } \\
0.86(0.51,1.45) \\
1.31(0.71,2.42) \\
0.65(0.38,1.09) \\
\end{array}$ & $\begin{array}{l}0.576 \\
0.389 \\
0.100 \\
\end{array}$ & $\begin{array}{l}45.0 \\
15.1 \\
23.4 \\
14.6\end{array}$ & $\begin{array}{l}55.0 \\
84.9 \\
76.6 \\
85.4 \\
\end{array}$ & $\begin{array}{c}\text { Ref } \\
0.22(0.12,0.40) \\
0.37(0.21,0.66) \\
0.21(0.11,0.40) \\
\end{array}$ & $\begin{array}{c}<0.001 \\
0.001 \\
<0.001 \\
\end{array}$ \\
\hline $\begin{array}{l}\text { Age (in years) } \\
<20 \\
20-29 \\
30-39 \\
\geq 40\end{array}$ & $\begin{array}{c}34 \\
319 \\
226 \\
22\end{array}$ & $\begin{array}{l}85.3 \\
74.3 \\
74.3 \\
72.7 \\
\end{array}$ & $\begin{array}{l}14.7 \\
25.7 \\
25.7 \\
27.3\end{array}$ & $\begin{array}{c}2.01(0.75,5.36) \\
\quad \operatorname{Ref} \\
1.00(0.68,1.48) \\
0.92(0.35,2.44)\end{array}$ & $\begin{array}{l}0164 \\
0.991 \\
0.871\end{array}$ & $\begin{array}{c}41.2 \\
35.1 \\
32.3 \\
9.1\end{array}$ & $\begin{array}{l}58.8 \\
64.9 \\
67.7 \\
90.9\end{array}$ & $\begin{array}{c}1.29(0.63,2.66) \\
\text { Ref } \\
0.88(0.61,1.27) \\
0.18(0.04,0.81)\end{array}$ & $\begin{array}{l}0.484 \\
0.495 \\
\mathbf{0 . 0 2 5}\end{array}$ \\
\hline $\begin{array}{l}\text { Ethnicity } \\
\text { Dagomba } \\
\text { Fulani } \\
\text { Others (e.g. Akan, Ewe } \\
\text { etc.) }\end{array}$ & $\begin{array}{c}576 \\
8 \\
15\end{array}$ & $\begin{array}{l}74.8 \\
87.5 \\
66.7\end{array}$ & $\begin{array}{l}25.2 \\
12.5 \\
33.3\end{array}$ & $\begin{array}{c}\text { Ref } \\
2.35(0.28,19.30) \\
0.67(0.23,2.00)\end{array}$ & $\begin{array}{l}0.425 \\
0.476\end{array}$ & $\begin{array}{l}33.8 \\
25.0 \\
20.0\end{array}$ & $\begin{array}{l}66.2 \\
75.0 \\
80.0\end{array}$ & $\begin{array}{c}\text { Ref } \\
0.65(0.13,3.26) \\
0.49(0.14,1.75)\end{array}$ & $\begin{array}{l}0.602 \\
0.271\end{array}$ \\
\hline $\begin{array}{l}\text { Education status } \\
\text { None } \\
\text { Primary } \\
\text { JSS/Middle School } \\
\text { Senior Secondary } \\
\text { Tertiary } \\
\end{array}$ & $\begin{array}{c}450 \\
46 \\
53 \\
38 \\
14 \\
\end{array}$ & $\begin{array}{l}76.2 \\
76.1 \\
69.8 \\
73.7 \\
50.0 \\
\end{array}$ & $\begin{array}{l}23.8 \\
23.9 \\
30.2 \\
26.3 \\
50.0 \\
\end{array}$ & $\begin{array}{c}\text { Ref } \\
0.99(0.49,2.02) \\
0.72(0.39,1.35) \\
0.87(0.41,1.86) \\
0.31(0.11,0.91) \\
\end{array}$ & $\begin{array}{l}0.984 \\
0.306 \\
0.725 \\
\mathbf{0 . 0 3 3} \\
\end{array}$ & $\begin{array}{l}30.9 \\
41.3 \\
41.5 \\
44.7 \\
28.6 \\
\end{array}$ & $\begin{array}{l}69.1 \\
58.7 \\
58.5 \\
55.3 \\
71.4 \\
\end{array}$ & $\begin{array}{c}\text { Ref } \\
1.57(0.85,2.93) \\
1.59(0.89,2.84) \\
1.81(0.93,3.54) \\
0.89(0.28,2.90) \\
\end{array}$ & $\begin{array}{l}0.151 \\
0.119 \\
0.082 \\
0.853 \\
\end{array}$ \\
\hline $\begin{array}{l}\text { Occupation status } \\
\text { Farmer } \\
\text { Petty trader } \\
\text { Salaried worker } \\
\text { Artisan/laborer } \\
\text { No occupation } \\
\text { Others }\end{array}$ & $\begin{array}{c}233 \\
181 \\
14 \\
79 \\
75 \\
19 \\
\end{array}$ & $\begin{array}{l}77.7 \\
70.2 \\
57.1 \\
77.2 \\
77.3 \\
78.9\end{array}$ & $\begin{array}{l}22.3 \\
29.8 \\
42.9 \\
22.8 \\
22.7 \\
21.1\end{array}$ & $\begin{array}{c}\text { Ref } \\
0.68(0.43,1.05) \\
0.38(0.13,1.15) \\
0.97(0.53,1.79) \\
0.98(0.53,1.83) \\
1.08(0.34,3.39) \\
\end{array}$ & $\begin{array}{l}0.083 \\
0.088 \\
0.931 \\
0.950 \\
0.899 \\
\end{array}$ & $\begin{array}{l}31.3 \\
29.8 \\
28.6 \\
34.2 \\
41.3 \\
63.2 \\
\end{array}$ & $\begin{array}{l}68.7 \\
70.2 \\
71.4 \\
65.8 \\
58.7 \\
36.8\end{array}$ & $\begin{array}{c}\text { Ref } \\
0.93(0.61,1.42) \\
0.88(0.27,2.89) \\
1.14(0.66,1.96) \\
1.54(0.90,2.64) \\
3.76(1.42,9.94) \\
\end{array}$ & $\begin{array}{l}0.743 \\
0.829 \\
0.640 \\
0.112 \\
\mathbf{0 . 0 0 8} \\
\end{array}$ \\
\hline $\begin{array}{l}\text { Wealth index } \\
\text { Poorest }\end{array}$ & 118 & 73.7 & 26.3 & $1.32(0.75,2.31)$ & 0.332 & 28.8 & 71.2 & $0.89(0.51,1.56)$ & 0.693 \\
\hline
\end{tabular}




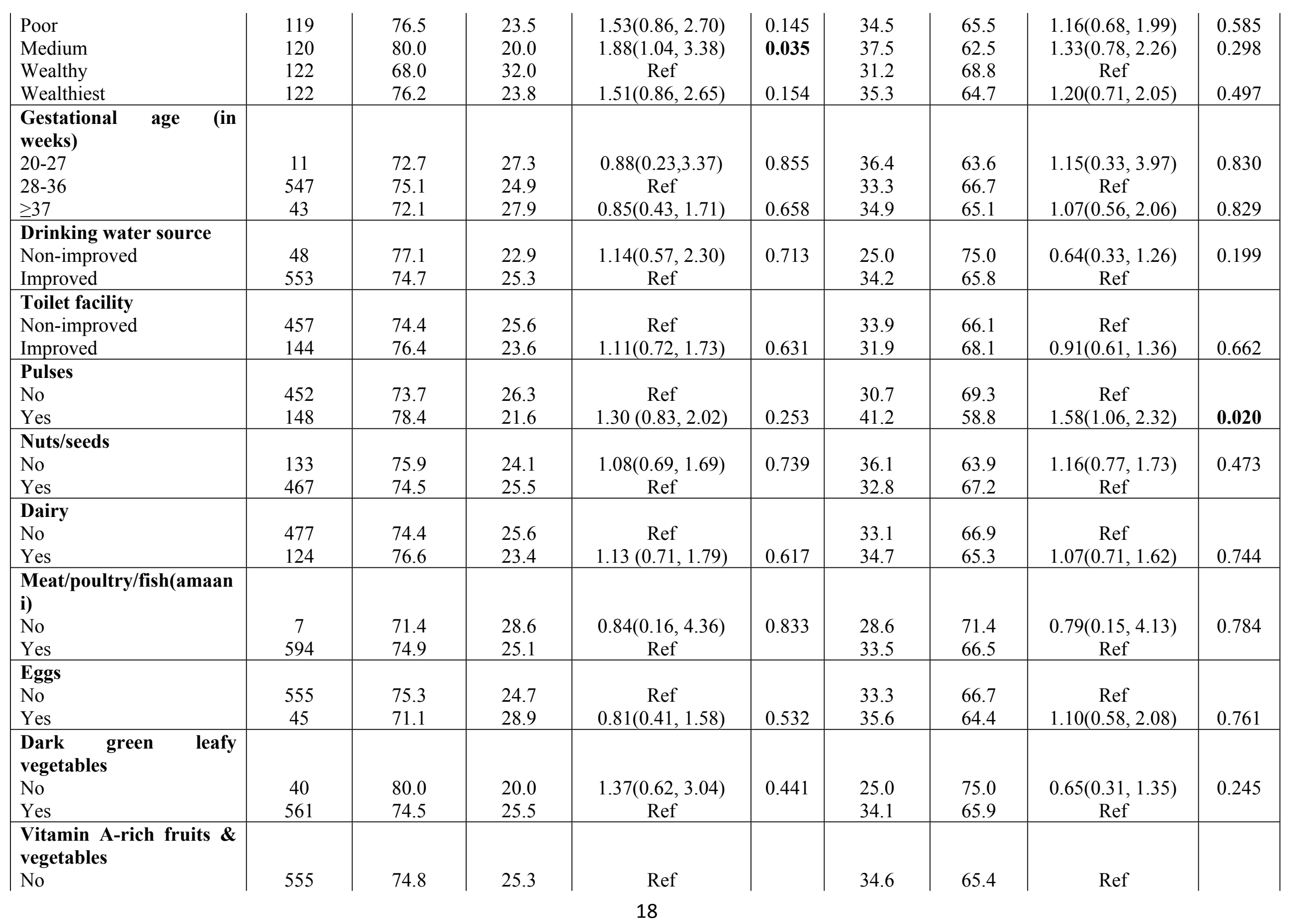




\begin{tabular}{|c|c|c|c|c|c|c|c|c|c|}
\hline Yes & 46 & 76.1 & 23.9 & $1.07(0.53,2.17)$ & 0.844 & 19.6 & 80.4 & $0.46(0.22,0.97)$ & 0.042 \\
\hline $\begin{array}{l}\text { Other vegetables } \\
\text { No } \\
\text { Yes }\end{array}$ & $\begin{array}{c}11 \\
590\end{array}$ & $\begin{array}{l}81.8 \\
74.7\end{array}$ & $\begin{array}{l}18.2 \\
25.3 \\
\end{array}$ & $\begin{array}{c}1.52(0.32,7.12) \\
\text { Ref }\end{array}$ & 0.595 & $\begin{array}{l}27.3 \\
33.6 \\
\end{array}$ & $\begin{array}{l}72.7 \\
66.4\end{array}$ & $\begin{array}{c}0.74(0.19,2.83) \\
\text { Ref }\end{array}$ & 0.663 \\
\hline $\begin{array}{l}\text { Other fruits } \\
\text { No } \\
\text { Yes }\end{array}$ & $\begin{array}{c}570 \\
30\end{array}$ & $\begin{array}{l}74.6 \\
80.0\end{array}$ & $\begin{array}{l}25.4 \\
20.0\end{array}$ & $\begin{array}{c}\operatorname{Ref} \\
1.36(0.54,3.40)\end{array}$ & 0.505 & $\begin{array}{l}33.5 \\
30.0\end{array}$ & $\begin{array}{l}66.5 \\
70.0\end{array}$ & $\begin{array}{c}\text { Ref } \\
0.85(0.38,1.89)\end{array}$ & 0.691 \\
\hline $\begin{array}{l}\text { Dietary diversity } \\
<5 \\
\geq 5\end{array}$ & $\begin{array}{c}91 \\
510\end{array}$ & $\begin{array}{l}73.6 \\
75.1\end{array}$ & $\begin{array}{l}26.4 \\
24.9\end{array}$ & $\begin{array}{c}0.93(0.56,1.54) \\
\text { Ref }\end{array}$ & 0.766 & $\begin{array}{l}29.7 \\
34.1\end{array}$ & $\begin{array}{l}70.3 \\
65.9\end{array}$ & $\begin{array}{c}0.81(0.50,1.32) \\
\text { Ref }\end{array}$ & 0.408 \\
\hline $\begin{array}{l}\text { Household livestock } \\
\text { No } \\
\text { Yes } \\
\end{array}$ & $\begin{array}{l}116 \\
485 \\
\end{array}$ & $\begin{array}{l}69.8 \\
76.1 \\
\end{array}$ & $\begin{array}{l}30.2 \\
23.9 \\
\end{array}$ & $\begin{array}{c}0.73(0.46,1.14) \\
\text { Ref }\end{array}$ & 0.164 & $\begin{array}{l}31.0 \\
34.0 \\
\end{array}$ & $\begin{array}{l}69.0 \\
66.0 \\
\end{array}$ & $\begin{array}{c}0.87(0.56,1.35) \\
\text { Ref }\end{array}$ & 0.540 \\
\hline $\begin{array}{l}\text { Household ITN } \\
\text { No } \\
\text { Yes } \\
\end{array}$ & $\begin{array}{c}55 \\
546 \\
\end{array}$ & $\begin{array}{l}80.0 \\
74.4 \\
\end{array}$ & $\begin{array}{l}20.0 \\
25.6 \\
\end{array}$ & $\begin{array}{c}1.38(0.69,2.74) \\
\text { Ref }\end{array}$ & 0.360 & $\begin{array}{l}49.1 \\
31.9 \\
\end{array}$ & $\begin{array}{l}50.9 \\
68.1 \\
\end{array}$ & $\begin{array}{c}2.06(1.18,3.60) \\
\operatorname{Ref}\end{array}$ & 0.011 \\
\hline $\begin{array}{l}\text { Slept under ITN previous } \\
\text { night } \\
\text { No } \\
\text { Yes }\end{array}$ & $\begin{array}{l}104 \\
497\end{array}$ & $\begin{array}{l}77.9 \\
74.3\end{array}$ & $\begin{array}{l}22.1 \\
25.7\end{array}$ & $\begin{array}{c}1.22(0.74,2.02) \\
\text { Ref }\end{array}$ & 0.437 & $\begin{array}{l}44.2 \\
31.2\end{array}$ & $\begin{array}{l}55.8 \\
68.8\end{array}$ & $\begin{array}{c}1.75(1.14,2.69) \\
\text { Ref }\end{array}$ & 0.011 \\
\hline $\begin{array}{l}\text { No. of previous deliveries } \\
0 \\
1 \\
2-3 \\
\geq 4\end{array}$ & $\begin{array}{l}142 \\
122 \\
214 \\
123\end{array}$ & $\begin{array}{l}81.7 \\
77.1 \\
70.1 \\
73.2\end{array}$ & $\begin{array}{l}18.3 \\
22.9 \\
29.9 \\
26.8\end{array}$ & $\begin{array}{c}1.90(1.14,3.19) \\
1.43(0.86,2.39) \\
\text { Ref } \\
1.16(0.71,1.91)\end{array}$ & $\begin{array}{l}\mathbf{0 . 0 1 5} \\
0.170 \\
0.548\end{array}$ & $\begin{array}{c}47.9 \\
32.8 \\
27.6 \\
27.64\end{array}$ & $\begin{array}{c}52.1 \\
67.2 \\
72.4 \\
72.36\end{array}$ & $\begin{array}{c}2.41(1.55,3.77) \\
1.28(0.79,2.08) \\
\text { Ref } \\
1.00(0.61,1.65)\end{array}$ & $\begin{array}{c}<\mathbf{0 . 0 0 1} \\
0.314 \\
0.989\end{array}$ \\
\hline $\begin{array}{l}\text { Total ANC visits } \\
<4 \\
4-7 \\
\geq 8\end{array}$ & $\begin{array}{c}286 \\
286 \\
29\end{array}$ & $\begin{array}{l}76.9 \\
72.4 \\
79.3\end{array}$ & $\begin{array}{l}23.1 \\
27.6 \\
20.7\end{array}$ & $\begin{array}{c}\operatorname{Ref} \\
0.79(0.54,1.15) \\
1.15(0.45,2.94)\end{array}$ & $\begin{array}{l}0.212 \\
0.771\end{array}$ & $\begin{array}{l}33.9 \\
33.6 \\
27.6\end{array}$ & $\begin{array}{l}66.1 \\
66.4 \\
72.4\end{array}$ & $\begin{array}{c}\text { Ref } \\
0.98(0.70,1.39) \\
0.74(0.32,1.74)\end{array}$ & $\begin{array}{l}0.930 \\
0.492\end{array}$ \\
\hline $\begin{array}{l}\text { IPT received } \\
\text { No } \\
\text { Yes } \\
\end{array}$ & $\begin{array}{c}99 \\
502 \\
\end{array}$ & $\begin{array}{l}79.8 \\
73.9 \\
\end{array}$ & $\begin{array}{l}20.2 \\
26.1\end{array}$ & $\begin{array}{c}1.39(0.82,2.37) \\
\text { Ref }\end{array}$ & 0.218 & $\begin{array}{l}41.4 \\
31.9 \\
\end{array}$ & $\begin{array}{l}58.6 \\
68.1 \\
\end{array}$ & $\begin{array}{c}1.51(0.97,2.35) \\
\text { Ref }\end{array}$ & 0.067 \\
\hline $\begin{array}{l}\text { Deworming medication } \\
\text { No } \\
\text { Yes }\end{array}$ & $\begin{array}{c}515 \\
86\end{array}$ & $\begin{array}{l}74.0 \\
80.2\end{array}$ & $\begin{array}{l}26.0 \\
19.8 \\
\end{array}$ & $\begin{array}{c}\operatorname{Ref} \\
1.43(0.81,2.51)\end{array}$ & 0.218 & $\begin{array}{l}35.5 \\
20.9\end{array}$ & $\begin{array}{l}64.5 \\
79.1\end{array}$ & $\begin{array}{c}\operatorname{Ref} \\
0.48(0.28,0.83)\end{array}$ & 0.009 \\
\hline
\end{tabular}


256 bivariate analysis (Table 3), tertiary education $(\mathbf{a O R}=\mathbf{0 . 2 0}, 95 \% \mathrm{CI}: 0.04,0.96)$ and no previous delivery

$257(\mathbf{a O R}=\mathbf{2 . 1 3}, 95 \% \mathrm{CI}: 1.18,3.85)$ were the only two factors that were significantly associated with anemia

$258(<11.0 \mathrm{~g} / \mathrm{dl})$ (Table 3). The current study found that maternal dietary diversity ( $<5 \mathrm{MDD}-\mathrm{W})$ was not 259 statistically associated with anemia (aOR=0.80, 95\%CI:0.67, 1.38) or moderate/severe anemia $260(\mathrm{aOR}=0.74,95 \% \mathrm{CI}: 0.44,1.24)$. However, in using moderate/severe anemia $(<9.0 \mathrm{~g} / \mathrm{dl})$ as the cut-off 261 point for anemia, three explanatory factors were significantly associated. That is, participants whose 262 occupation status was categorized as "other" (e.g. herdsmen) were three times more likely to have 263 moderate/severe anemia (aOR=2.90, 95\%CI: 1.04, 8.09). Women who had no previous delivery 264 continued to be more likely to have moderate/severe anemia (aOR=2.13, 95\%CI: 1.28, 3.54). Finally, 265 participants who received deworming medication remained at lower risk of moderate/severe anemia $266(\mathbf{a O R}=\mathbf{0 . 5 1}, 95 \% \mathrm{CI}: 0.29,0.90)$ than those who did not take these medications. 
Table 3: Multivariate analysis of anemia status among pregnant women in Northern Ghana $(n=601)$

\begin{tabular}{|c|c|c|c|c|c|c|c|c|c|}
\hline & & $\begin{array}{c}\text { Anemia } \\
(<11.0 \mathrm{~g} / \mathrm{dl})\end{array}$ & $\begin{array}{l}\text { No anemia } \\
(\geq 11.0 \mathrm{~g} / \mathrm{dl})\end{array}$ & $\begin{array}{l}\text { Anemia }(<11.0 \mathrm{~g} / \mathrm{c} \\
\text { versus no anemia } \\
(\geq 11.0 \mathrm{~g} / \mathrm{dl})\end{array}$ & & $\begin{array}{c}\text { Moderate/severe } \\
\text { anemia } \\
(<9.0 \mathrm{~g} / \mathrm{dl}) \\
\end{array}$ & $\begin{array}{l}\text { Mild/no } \\
\text { anemia } \\
(\geq 9.0 \mathrm{~g} / \mathrm{dl})\end{array}$ & $\begin{array}{r}\text { Moderate/severe } \\
(<9.0 \mathrm{~g} / \mathrm{dl}) \text { versus } \\
\text { anemia }(\geq 9.0 \\
\end{array}$ & $\begin{array}{l}\text { nemia } \\
\text { iild/no } \\
\text { dl) }\end{array}$ \\
\hline & Frequency & $\%$ & $\%$ & Adjusted OR(CI) & $\begin{array}{c}\mathrm{P}- \\
\text { value }\end{array}$ & $\%$ & $\%$ & Adjusted OR(CI) & $\begin{array}{c}\text { P- } \\
\text { value }\end{array}$ \\
\hline $\begin{array}{l}\text { Education status } \\
\text { None } \\
\text { Primary } \\
\text { JSS/Middle School } \\
\text { Senior Secondary } \\
\text { Tertiary }\end{array}$ & $\begin{array}{c}450 \\
46 \\
53 \\
38 \\
14 \\
\end{array}$ & $\begin{array}{c}76.2 \\
76.1 \\
69.8 \\
73.7 \\
50.00 \\
\end{array}$ & $\begin{array}{c}23.8 \\
23.9 \\
30.2 \\
26.3 \\
50.00 \\
\end{array}$ & $\begin{array}{c}\text { Ref } \\
0.96(0.45,2.03) \\
0.59(0.30,1.18) \\
0.64(0.27,1.52) \\
\mathbf{0 . 2 0}(0.04,0.96) \\
\end{array}$ & $\begin{array}{l}0.908 \\
0.135 \\
0.312 \\
\mathbf{0 . 0 4 4} \\
\end{array}$ & $\begin{array}{l}30.9 \\
41.3 \\
41.5 \\
44.7 \\
28.6 \\
\end{array}$ & $\begin{array}{l}69.1 \\
58.7 \\
58.5 \\
55.3 \\
71.4 \\
\end{array}$ & $\begin{array}{c}\text { Ref } \\
1.48(0.76,2.88) \\
1.31(0.69,2.49) \\
1.19(0.54,2.62) \\
0.75(0.15,3.71) \\
\end{array}$ & $\begin{array}{l}0.253 \\
0.407 \\
0.657 \\
0.723 \\
\end{array}$ \\
\hline $\begin{array}{l}\text { Occupation status } \\
\text { Farmer } \\
\text { Petty trader } \\
\text { Salaried worker } \\
\text { Artisan/laborer } \\
\text { No occupation } \\
\text { Others } \\
\end{array}$ & $\begin{array}{c}233 \\
181 \\
14 \\
79 \\
75 \\
19 \\
\end{array}$ & $\begin{array}{l}77.7 \\
70.2 \\
57.1 \\
77.2 \\
77.3 \\
78.9\end{array}$ & $\begin{array}{l}22.3 \\
29.8 \\
42.9 \\
22.8 \\
22.7 \\
21.1\end{array}$ & $\begin{array}{c}\text { Ref } \\
0.72(0.45,1.17) \\
0.85(0.16,4.46) \\
0.95(0.48,1.89) \\
0.93(0.47,1.86) \\
1.17(0.35,3.87) \\
\end{array}$ & $\begin{array}{l}0.185 \\
0.852 \\
0.874 \\
0.844 \\
0.797 \\
\end{array}$ & $\begin{array}{l}31.3 \\
29.8 \\
28.6 \\
34.2 \\
41.3 \\
63.2 \\
\end{array}$ & $\begin{array}{l}68.7 \\
70.2 \\
71.4 \\
65.8 \\
58.7 \\
36.8 \\
\end{array}$ & $\begin{array}{c}\text { Ref } \\
0.95(0.60,1.51) \\
0.95(0.19,4.72) \\
0.83(0.45,1.53) \\
1.32(0.72,2.41) \\
\mathbf{2 . 9 0 ( 1 . 0 4 , 8 . 0 9 )} \\
\end{array}$ & $\begin{array}{l}0.840 \\
0.948 \\
0.549 \\
0.367 \\
\mathbf{0 . 0 4 2} \\
\end{array}$ \\
\hline $\begin{array}{l}\text { Wealth index } \\
\text { Poorest } \\
\text { Poor } \\
\text { Medium } \\
\text { Wealthy } \\
\text { Wealthiest }\end{array}$ & $\begin{array}{l}118 \\
119 \\
120 \\
122 \\
122\end{array}$ & $\begin{array}{l}73.7 \\
76.5 \\
80.0 \\
68.0 \\
76.2 \\
\end{array}$ & $\begin{array}{l}26.3 \\
23.5 \\
20.0 \\
32.0 \\
23.8\end{array}$ & $\begin{array}{c}1.14(0.62,2.08) \\
1.40(0.77,2.54) \\
1.80(0.98,3.31) \\
\text { Ref } \\
1.65(0.90,3.01)\end{array}$ & $\begin{array}{l}0.676 \\
0.277 \\
0.059 \\
0.102 \\
\end{array}$ & $\begin{array}{l}28.8 \\
34.5 \\
37.5 \\
31.2 \\
35.3 \\
\end{array}$ & $\begin{array}{l}71.2 \\
65.5 \\
62.5 \\
68.8 \\
64.7 \\
\end{array}$ & $\begin{array}{c}0.96(0.52,1.75) \\
1.33(0.74,2.38) \\
1.46(0.83,2.57) \\
\text { Ref } \\
1.20(0.68,2.12)\end{array}$ & $\begin{array}{l}0.893 \\
0.337 \\
0.191 \\
\\
0.533 \\
\end{array}$ \\
\hline $\begin{array}{l}\text { Gestational age (in } \\
\text { weeks) } \\
20-27 \\
28-36 \\
\geq 37 \\
\end{array}$ & $\begin{array}{c}11 \\
547 \\
43 \\
\end{array}$ & $\begin{array}{l}72.7 \\
75.1 \\
72.1\end{array}$ & $\begin{array}{l}27.3 \\
24.9 \\
27.9 \\
\end{array}$ & $\begin{array}{c}1.17(0.28,4.89) \\
\text { Ref } \\
0.63(0.28,1.41) \\
\end{array}$ & $\begin{array}{l}0.830 \\
0.261 \\
\end{array}$ & $\begin{array}{l}36.4 \\
33.3 \\
34.9 \\
\end{array}$ & $\begin{array}{l}63.6 \\
66.7 \\
65.1 \\
\end{array}$ & $\begin{array}{c}1.23(0.34,4.54) \\
\operatorname{Ref} \\
1.25(0.58,2.68)\end{array}$ & $\begin{array}{l}0.752 \\
0.572 \\
\end{array}$ \\
\hline $\begin{array}{l}\text { Dietary diversity } \\
<5 \\
\geq 5\end{array}$ & $\begin{array}{c}91 \\
510\end{array}$ & $\begin{array}{l}73.6 \\
75.1\end{array}$ & $\begin{array}{l}26.4 \\
24.9 \\
\end{array}$ & $\begin{array}{c}0.80(0.47,1.38) \\
\text { Ref }\end{array}$ & 0.427 & $\begin{array}{l}29.7 \\
34.1 \\
\end{array}$ & $\begin{array}{l}70.3 \\
65.9 \\
\end{array}$ & $\begin{array}{c}0.74(0.44,1.24) \\
\text { Ref }\end{array}$ & 0.249 \\
\hline $\begin{array}{l}\text { Slept under ITN } \\
\text { previous night } \\
\text { No } \\
\text { Yes } \\
\end{array}$ & $\begin{array}{l}104 \\
497 \\
\end{array}$ & $\begin{array}{l}77.9 \\
74.3 \\
\end{array}$ & $\begin{array}{l}22.1 \\
25.7\end{array}$ & $\begin{array}{c}1.15(0.67,1.96) \\
\text { Ref }\end{array}$ & 0.621 & $\begin{array}{l}44.2 \\
31.2 \\
\end{array}$ & $\begin{array}{l}55.8 \\
68.8 \\
\end{array}$ & $\begin{array}{c}1.48(0.93,2.35) \\
\text { Ref }\end{array}$ & 0.101 \\
\hline
\end{tabular}




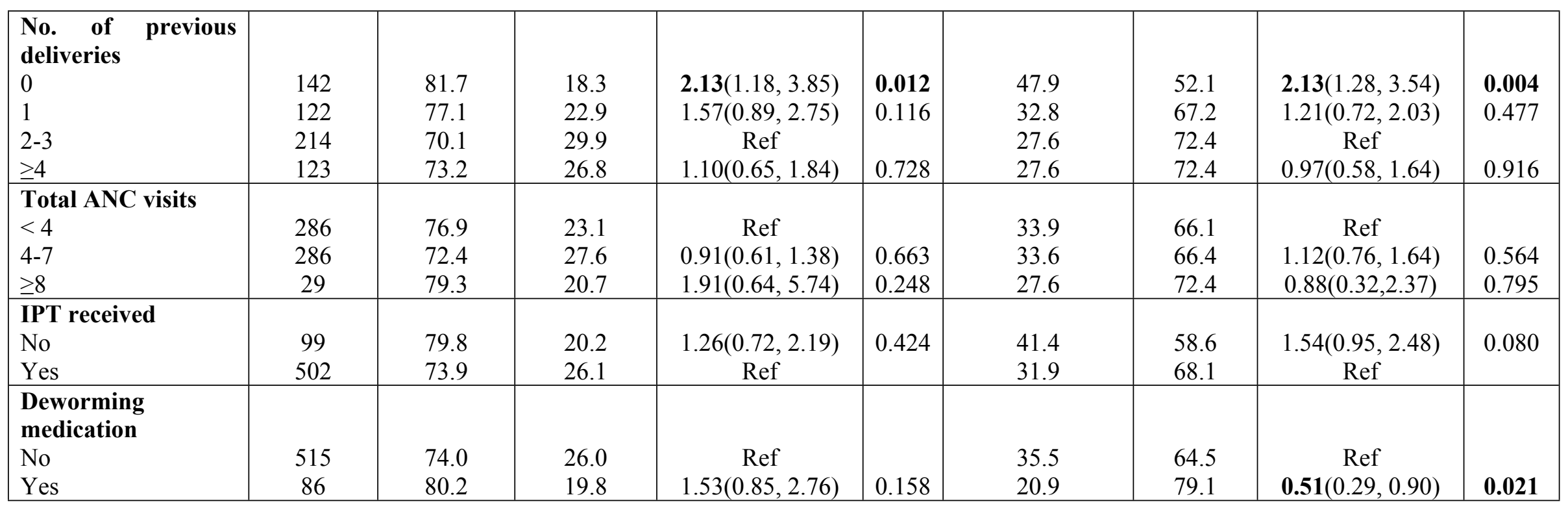




\section{DISCUSSION}

We sought to assess the independent contributions of dietary diversity and other predictors to anemia status during pregnancy. Three-quarters of women were anemic $(<11.0 \mathrm{~g} / \mathrm{dl})$, including one-third

of whom had moderate/severe anemia. This prevalence of anemia in our study area is considered by WHO to be a severe public health problem ${ }^{3}$, and is higher than that for Ghana as a whole, and for the

Africa 3,7,20,21,22. Not surprisingly, anemia prevalence among pregnant women varies by geographical area, culture, seasonality and countries ${ }^{23}$ such as $75 \%$ in the current study, compared with $18 \%$ and $57 \%$

in Ethiopia ${ }^{24,25}$. The high prevalence that we observed might be due to increased physiological demands

for nutrients during the second and third trimesters of pregnancy ${ }^{26}$. It could also be attributed to

addition to these possible explanations, the specific causes underlying the high anemia prevalence in the northern Ghana with prevalences of $47 \%$ mild, $20 \%$ moderate, and $3 \%$ severe ${ }^{10}$.

odds of moderate/severe anemia among pregnant women, while others such as occupation (e.g. herdsmen) and parity (primigravidae) were associated with higher odds of moderate/severe anemia. Our may be due to the experience acquired by women during previous pregnancies and with ANC services, 
mean $\mathrm{Hb}$ concentrations of $9.7 \mathrm{~g} / \mathrm{dl}$ for primigravidae, $10.1 \mathrm{~g} / \mathrm{dl}$ for secundigravidae and $10.5 \mathrm{~g} / \mathrm{dl}$ for multigravidae ${ }^{28}$. However, other studies have found that higher parity (multigravidae) was associated with greater risk of anemia $25,29,30,31$. This might be due to differences in birth spacing rather than total parity. For example, pregnant women with short pregnancy intervals were more likely to develop anemia during pregnancy than those with longer birth spacing ${ }^{27}$.

Our results indicated that pregnant women who worked in other occupations (e.g. herdsmen) often from minority ethnic groups, hence are more likely to delay ANC initiation until second or third trimester of pregnancy. Thus, they fail to exploit all opportunities offered by the ANC ${ }^{32}$. Salaried workers and traders had lower odds of moderate/severe anemia when compared to farmers. This may be due to their increased access to food, greater dietary diversity and improved food security compared to farmers, for example, whose livelihood depends on seasonal crops to meet their nutrient requirements. moderate/severe anemic. This is consistent with previous studies where primigravidae had increased risk 311 of anemia compared to multigravidae ${ }^{12}$. Until uncertain rituals are performed by the family members of 312 the husband to publicly declare or announce the pregnancy, they are unwilling to start ANC visits for 313 fear of miscarriage or other complications. 
319 associated with anemia and MDD-W was not associated with anemia ${ }^{10}$, but another study found that

320 high maternal MDD-W was associated with a decreased risk of anemia ${ }^{20}$. Diet is an important factor for

321 anemia. Some eating patterns or habits may predispose pregnant women to an increased risk of

322 developing anemia. Poor dietary diversity leads to inadequacies in minerals and vitamins. Though we

323 observed that $85 \%$ of the pregnant women consumed $\geq 5$ food groups in the previous day, dietary

324 diversity was not associated with a higher Hb concentration. This lack of association between MDD-W

325 and moderate/severe anemia may be due to inappropriate kinds of foods (i.e. animal-source foods with

326 highly bioavailable iron) that would be linked to lower anemia risk or challenges of nutrients

327 bioavailability from the consumed diets. We also found that consumption of animal-source foods was

328 not associated with lower odds of anemia in the current study.

329 Overall, $91 \%$ of pregnant women reported of ITN ownership and $83 \%$ slept under the ITN the

330 night before the interview. The coverage and utilization of ITN higher than a previous study conducted

331 in southern Ghana where $75 \%$ of pregnant women reported ownership of ITN and only $49 \%$ slept under

332 it the night preceding enrolment ${ }^{33}$. Similarly, the coverage of at least one IPT dose (84\%) in the current

333 study exceeds the national average $(68 \%)^{33}$. Independent of malaria interventions (household ownership

334 of ITN and slept under ITN on previous night), $\geq 4$ ANC visits and deworming medication were

335 associated with lower odds of moderate/severe anemia among pregnant women aged $\geq 20$ weeks of

336 gestation. In our study, deworming medication was protective against moderate/severe anemia. This

337 varies from the a previous study which reported that iron supplementation and deworming treatment was

338 not significant associated with anemia ${ }^{30}$. We observed that pregnant women who received IPT $(83 \%)$

339 had lesser of risk to develop moderate/severe anemia. An explanatory factor such as IPT was not

340 associated with moderate/severe anemia. Our current finding is similar to a study conducted in Ghana

341 which found that IPT shown a weak association with anemia using bivariate analysis but no association

342 when adjusted for confounders in multivariate analysis ${ }^{20}$. In a previous study conducted in Ethiopia, 
343 malaria infection during pregnancy had higher risk to develop anemia ${ }^{27}$. Our study found that ownership

344 of ITN and utilization of ITN were associated with reduced odds of moderate/severe anemia. This is

345 suggestive of effective intervention in the study area. Deworming medication was associated with

346 moderate/severe anemia. Parasitic infections are known to be a major cause of anemia. Thus, treatment

347 against intestinal parasites help to improve hemoglobin concentration ${ }^{3}$.

348

\section{Strengths and Limitations}

350

In terms of strength, the study focused on an important category of pregnant women with

351 implications on birth outcomes. The sample size was also large and representative of the population

352 accessing ANC services at HFs. This is probably the first study to assess the predicators of

353 moderate/severe anemia among pregnant women ( $\geq 20$ gestational weeks) in northern Ghana.

354 This study had several limitations and the findings should be interpreted as such. The study

355 design was cross-sectional and does not allow us to assess causality. In addition, participant recall bias

356 was a limitation but the interviews were conducted using a structured questionnaire and the interviewers

357 were trained nurses and health science educators to mitigate the effect of recall bias. The study sample

358 was limited to pregnant women who received ANC services at HFs, thus, some pregnant women were

359 excluded. This may not be reflective of the study setting. However, the majority of Ghanaian women 360 now seek ANC services.

The prevalence of anemia among pregnant women was nearly twice as national estimate in

364 Ghana, and varied by maternal age, parity and occupation. Minimum dietary diversity was not associated 
366 associated with moderate/severe anemia. There is the need to intensify education on ANC services, ITN

367 utilization and deworming medication to prevent and reduce anemia prevalence among pregnant women.

\section{Acknowledgements}

We are grateful to the two research assistants and staff of Ghana Health Service who

\section{Data Availability Statement}

The dataset used and/or analyzed during the study is available from the corresponding author upon request.

\section{Author contributions}

MNA and MW conceptualized and designed the protocol and received support from AJ

MNA and MW drafted the manuscript. RA, AJ and MY edited the manuscript. All authors read and approved the final manuscript.

\section{REFERENCES}

1. Kassa, G. M., Muche, A. A., Berhe, A. K. \& Fekadu, G. A. Prevalence and determinants of anemia among pregnant women in Ethiopia ; a systematic review and meta-analysis. 1-9 (2017). doi:10.1186/s12878-017-0090-z

2. Black, R. E. et al. Maternal and Child Nutrition 1 Maternal and child undernutrition and overweight in low-income and middle-income countries. (2011). doi:10.1016/S0140-6736(13)60937-X

3. WHO. The global prevalence of anaemia in 2011. Geneva: World Health Organization. WHO Report (2015). doi:10.1017/S1368980008002401

4. $\quad$ Engmann, C., Adanu, R., Lu, T., Bose, C. \& Lozoff, B. Anemia and iron deficiency in pregnant Ghanaian women from urban areas. 62-66 (2008). doi:10.1016/j.ijgo.2007.09.032

5. WHO. WHO Recommendation on Antenatal care for positive pregnancy experience. WHO Recomm. Antenatal care Posit. pregnancy Exp. (2016). 
409

410

411

412

413

414

415

416

417

418

419

420

421

422

423

424

425

426

427

428

429

430

431

432

433

434

435

436

437

438

439

440

441

442

443

444

445

446

447

448

6. Tadesse, S. E. et al. Determinants of anemia among pregnant mothers attending antenatal care in Dessie town health facilities , northern central Ethiopia , unmatched case -control study. 1-9 (2017). doi:10.1371/journal.pone. 0173173

7. GSS, GHS \& ICF. International. Ghana Demographic and Health Survey 2014. Rockville, Maryland, USA: Ghana Statitiscal Service, Ghana Health Service, and ICF International. (2015).

8. WHO. Worldwide prevalence of anaemia 1993-2005: WHO Global Database on Anaemia. World Health Organization. (2008).

9. Ngnie-teta, I., Kuate-defo, B. \& Receveur, O. Multilevel modelling of sociodemographic predictors of various levels of anaemia among women in Mali. 12, 1462-1469 (2008).

10. Saaka, M., Oladele, J., Larbi, A. \& Hoeschle-zeledon, I. Dietary Diversity Is Not Associated with Haematological Status of Pregnant Women Resident in Rural Areas of Northern Ghana. 2017, (2017).

11. Kumar, A., Rai, A. K., Basu, S., Dash, D. \& Singh, J. S. Cord Blood and Breast Milk Iron Status in. 121, (2008).

12. Munasinghe, S. \& Broek, N. Van Den. Anaemia In Pregnancy In Malawi- A Review. 18, 160-174 (2006).

13. Darnton-hill, I. \& Mkparu, U. C. Micronutrients in Pregnancy in Low- and Middle-Income Countries. 1744-1768 (2015). doi:10.3390/nu7031744

14. Scholl, T. O. Iron status during pregnancy : setting the stage for mother and iron deficiency in women. 81, 1218-1222 (2018).

15. Zerfu, T. A., Umeta, M. \& Baye, K. Dietary diversity during pregnancy is associated with reduced risk of maternal anemia , preterm delivery, and low birth weight in a prospective cohort study in rural Ethiopia 1. 2011, 1482-1488 (2016).

16. Ghose, B. \& Yaya, S. Fruit and vegetable consumption and anemia among adult non-pregnant women : Ghana Demographic and Health Survey. 1-16 (2018). doi:10.7717/peerj.4414

17. Desta, M., Akibu, M., Tadese, M. \& Tesfaye, M. Dietary Diversity and Associated Factors among Pregnant Women Attending Antenatal Clinic in Shashemane, Oromia , Central Ethiopia : A CrossSectional Study. 2019, 7-10 (2019).

18. Scholl, T. O. Iron status during pregnancy: setting the stage for mother and infant. Am J Clin Nutr 81, (2005).

19. FAO. Minimum Dietary Diversity for Women A Guide to Measurement. The Food and Agriculture Organization of the United Nations and USAID's Food and Nutrition Technical Assistance III Project, Rome. (2016).

20. Saaka, M. \& Abdul, A. Role dietary diversity in ensuring adequate haematological status during pregnancy. 4, 749-755 (2015).

21. Tay, S. C. K., Agboli, E., Abruquah, H. H. \& Walana, W. Malaria and Anaemia in Pregnant and NonPregnant Women of Child-Bearing Age at the University Hospital ,. 2013, 193-200 (2013).

22. Anlaakuu, P. et al. Anaemia in pregnancy and associated factors : a cross sectional study of antenatal attendants at the Sunyani Municipal Hospital, Ghana. Malar. J. 71, 1280-1284 (2017).

23. Chowdhury, H. A. et al. Factors associated with maternal anaemia among pregnant women in Dhaka city. BMC Womens. Health 15, 1-6 (2015).

24. Alene, K. A., Dohe, A. M. \& Mohamed Dohe, A. Prevalence of Anemia and Associated Factors among Pregnant Women in an Urban Area of Eastern Ethiopia. Anemia 2014, (2015).

25. Stephen, G. et al. Anaemia in Pregnancy : Prevalence, Risk Factors, and Adverse Perinatal Outcomes in Northern Tanzania. 2018, (2018).

26. Lebso, M., Anato, A. \& Loha, E. Prevalence of anemia and associated factors among pregnant women in Southern Ethiopia: A community based cross-sectional study. 1-11 (2017). doi:10.1371/journal.pone.0188783

27. Kassa, G. M., Muche, A. A., Berhe, A. K. \& Fekadu, G. A. Prevalence and determinants of anemia among pregnant women in Ethiopia; a systematic review and meta-analysis. BMC Hematol. 17, 1-9 (2017).

28. Ofori, M. F., Ansah, E., Agyepong, I., Hviid, L. \& Akanmori, B. D. Pregnancy associated malaria in a rural community of Ghana. 43, (2009). 
449

450

451

452

453

454

455

456

457

458

459

460

461

462

29. Al-Shafei, M. A. et al. Effect of high parity on the occurrence of prediabetes: a cohort study. Acta Obstet. Gynecol. Scand. 89, 1182-1186 (2010).

30. Zillmer, K. et al. Predictors of anemia in pregnant women residing in rural areas of the Oromiya region of Ethiopia. 1-8 (2017). doi:10.1186/s40795-017-0166-y

31. Dar, B. Pregnant mothers are more anemic than lactating mothers, a comparative. 1-7 (2018). doi:10.1186/s12878-018-0096-1

32. Begum, K. et al. Prevalence of and factors associated with antenatal care seeking and adherence to recommended iron - folic acid supplementation among pregnant women in Zinder , Niger. 14, 1-11 (2018).

33. Ampofo, G. D., Tagbor, H. \& Bates, I. Effectiveness of pregnant women' s active participation in their antenatal care for the control of malaria and anaemia in pregnancy in Ghana : a cluster randomized controlled trial. Malar. J. 1-15 (2018). doi:10.1186/s12936-018-2387-1 